\title{
Article
}

\section{TaWAK2A-800, a Wall-Associated Kinase, Participates Positively in Resistance to Fusarium Head Blight and Sharp Eyespot in Wheat}

\author{
Feilong Guo ${ }^{1,2, \dagger}$, Tianci $\mathrm{Wu}^{1,2, \dagger}$, Gangbiao Xu ${ }^{2, \dagger}$, Haijun Qi ${ }^{1}$, Xiuliang Zhu ${ }^{1}$ and Zengyan Zhang ${ }^{1, *}$ \\ 1 The National Key Facility for Crop Gene Resources and Genetic Improvement, Institute of Crop Sciences, \\ Chinese Academy of Agricultural Sciences, Beijing 100081, China; guofeilong1117@163.com (F.G.); \\ wtc19961110@163.com (T.W.); haijunqi@yeah.net (H.Q.); zhuxiuliang@caas.cn (X.Z.) \\ 2 The Laboratory of Forestry Genetics, College of Forestry, Central South University of Forestry and \\ Technology, Changsha 410004, China; gangbiaoxu@163.com \\ * Correspondence: zhangzengyan@caas.cn \\ + These authors contributed equally to this work.
}

check for updates

Citation: Guo, F.; Wu, T.; Xu, G.; Qi, H.; Zhu, X.; Zhang, Z. TaWAK2A-800, a Wall-Associated Kinase, Participates Positively in Resistance to Fusarium Head Blight and Sharp Eyespot in Wheat. Int. J. Mol. Sci. 2021, 22, 11493. https://doi.org/10.3390/ ijms222111493

Academic Editors: Agata Gadaleta, Ilaria Marcotuli and Domenica Nigro

Received: 23 August 2021

Accepted: 29 September 2021

Published: 25 October 2021

Publisher's Note: MDPI stays neutral with regard to jurisdictional claims in published maps and institutional affiliations.

Copyright: (C) 2021 by the authors Licensee MDPI, Basel, Switzerland. This article is an open access article distributed under the terms and conditions of the Creative Commons Attribution (CC BY) license (https:// creativecommons.org/licenses/by/ $4.0 /)$.

\begin{abstract}
Fusarium head blight (FHB) and sharp eyespot are important diseases of the cereal plants, including bread wheat (Triticum aestivum) and barley. Both diseases are predominately caused by the pathogenic fungi, Fusarium graminearum and Rhizoctonia cerealis. The roles of the wheat-wallassociated kinases (WAKs) in defense against both $F$. graminearum and $R$. cerealis have remained largely unknown. This research reports the identification of TaWAK2A-800, a wheat WAK-coding gene located on chromosome $2 \mathrm{~A}$, and its functional roles in wheat resistance responses to FHB and sharp eyespot. TaWAK2A-800 transcript abundance was elevated by the early infection of $R$. cerealis and F. graminearum, or treatment with exogenous chitin. The gene transcript seemed to correspond to the resistance of wheat. Further functional analyses showed that silencing TaWAK2A-800 compromised the resistance of wheat to both FHB (F. graminearum) and sharp eyespot (R. cerealis). Moreover, the silencing reduced the expression levels of six defense-related genes, including the chitin-triggering immune pathway-marker genes, TaCERK1, TaRLCK1B, and TaMPK3. Summarily, TaWAK2A-800 participates positively in the resistance responses to both $F$. graminearum and $R$. cerealis, possibly through a chitin-induced pathway in wheat. TaWAK2A-800 will be useful for breeding wheat varieties with resistance to both FHB and sharp eyespot.
\end{abstract}

Keywords: bread wheat (Triticum aestivum); chitin-induced immunity; Fusarium graminearum; Rhizoctonia cereal; wall-associated kinase (WAK)

\section{Introduction}

Bread wheat (Triticum aestivum) is one of the most important staple food crops in the world. However, wheat production is threatened in many areas by devastating pests and pathogens [1]. Fusarium head blight (FHB) in bread wheat or barley is caused by a complex of Fusarium fungi, which may be dominated by Fusarium graminearum [2]. FHB can lead to marked yield losses [3]. Moreover, cereal grains infected with the Fusarium species may contain high levels of mycotoxins, which threaten the health of human beings and animals [4]. Rhizoctonia cerealis is a necrotrophic fungal pathogen responsible for the sharp eyespot, which is a devastating disease of wheat and other cereal plants in some countries of the world, especially in China [5,6]. The breeding of disease-resistant wheat varieties is the most environmentally sustainable and effective strategy for minimizing the losses caused by FHB and sharp eyespot. Unfortunately, traditional resistance breeding is challenging since there are no wheat cultivars with full resistance to FHB or sharp eyespot $[7,8]$. To improve wheat resistance to F. graminearum and $R$. cerealis, it is important to isolate the pivotal resistance QTL/genes. 
In plants, receptor-like kinases (RLKs) are involved in various life processes, such as growth, development, plant-pathogen interactions, and responses to environmental stimuli [9]. As a class of plant-specific RLKs, the protein sequences of wall-associated kinases (WAKs) and WAK-like (WAKLs) typically contain an aminol (N)-terminal signal peptide, an extracellular WAK-galacturonan-binding domain, an extracellular epidermal growth factor (EGF)-like domain, an EGF-calcium-binding (EGF-Ca ${ }^{2+}$ ) domain, a transmembrane domain, and a carboxyl (C)-terminal cytosolic serine/threonine kinase domain [10]. The extracellular EGF domain of WAKs has been determined to bind to the cell wall and persists during the process of plasmolysis [11]. Increasing evidence reveals that in several plant species, WAK/WAKL genes play an important role in plant-pathogen interactions. For instance, in Arabidopsis thaliana, the WAK gene, AtWAKL22, participates in resistance to Fusarium oxysporm [12]. In corn (Zea mays), qHSR1 and Htn1, encoding two distinct WAK proteins, mediate the resistance of corn to head-smut disease and northern corn leaf blight, respectively $[13,14]$. In rice (Oryza sativa), the WAK-encoding gene, Xa4's, high-expression improves resistance to bacterial blight disease [15]. In wheat, the WAK-encoding gene Stb6, isolated by the map-based cloning method, confers wheat resistance to Septoria tritici blotch disease caused by Zymoseptoria tritici [16]. Additionally, TaWAK6, another wheat WAK gene located on chromosome $5 \mathrm{~B}$, participates in adult plant resistance against wheat leaf rust [17]. Recently, Gadaleta et al. (2019) cloned an FHB-QTL based on map, located on the $2 \mathrm{~A}$ chromosome, and introgressed from the Chinese wheat cultivar, Sumai 3, from durum wheat, and it corresponded to the sequence with TraesCS2A02G071800.1 (hereafter named TaWAK2A-800). The relative transcript level of the TaWAK2A-800 gene in FHB-resistant wheat lines was significantly higher than in the susceptible wheat lines. After inoculation with F. graminearum, three TILLING mutant lines (Kronos3340, Kronos4209, Kronos3965) presented more severe FHB symptoms [18]. However, they did not demonstrate that TaWAK2A-800 might have potential broad-spectrum resistance traits in wheat against other pathogens.

To explore if TaWAK2A-800 participates in broad-spectrum resistance against both fungal pathogens, comparative transcriptomics and reverse transcription quantitative PCR (qRT-PCR) were used to test the TaWAK2A-800 gene transcript profiles in the wheat response to $R$. cerealis. Moreover, we further dissected the functional role of TaWAK2A800 in wheat defense against $R$. cerealis and F. graminearum infection. After $R$. cerealis and $F$. graminearum infection, TaWAK2A-800 was significantly induced in the $R$. cerealisresistant wheat cultivar, CI12633, and the F. graminearum-resistant cultivar, Sumai 3 . The functional assay results show that the expressed TaWAK2A-800 is required not only for wheat resistance to both sharp eyespot and FHB, but also for the expression of the receptorlike kinase-coding gene, TaCERK1, the wheat receptor-like cytoplasmic kinase-coding TaRLCK1B, and the wheat mitogen-activated protein kinase-coding TaMPK3, defensin, chitinase3, and $\beta-1,3$-glucanase in wheat. This study reveals the function of TaWAK2A-800 in the broad-spectrum resistance of wheat to both sharp eyespot by R. cerealis, and FHB by F. graminearum.

\section{Results}

\subsection{Transcript Abundance of TaWAK2A-800 Seems to Link to Wheat Resistance Degree of} $R$. cerealis and F. graminearum

On the basis of the RNA-sequencing data from sharp-eyespot-resistant and -susceptible recombinant inbred lines (RILs, cross of Shanhongmai and Wenmai 6), inoculation with the $R$. cerealis strain, RC207, at 4 and 10 days, and also mock, the gene, TaWAK2A-800 (sequence with ID TraesCS2A02G071800.1), was significantly higher in the resistant RIL pool (RIL-R) than in the susceptible RIL pool (RIL-S) at the same inoculation time points (Figure 1A). 
A
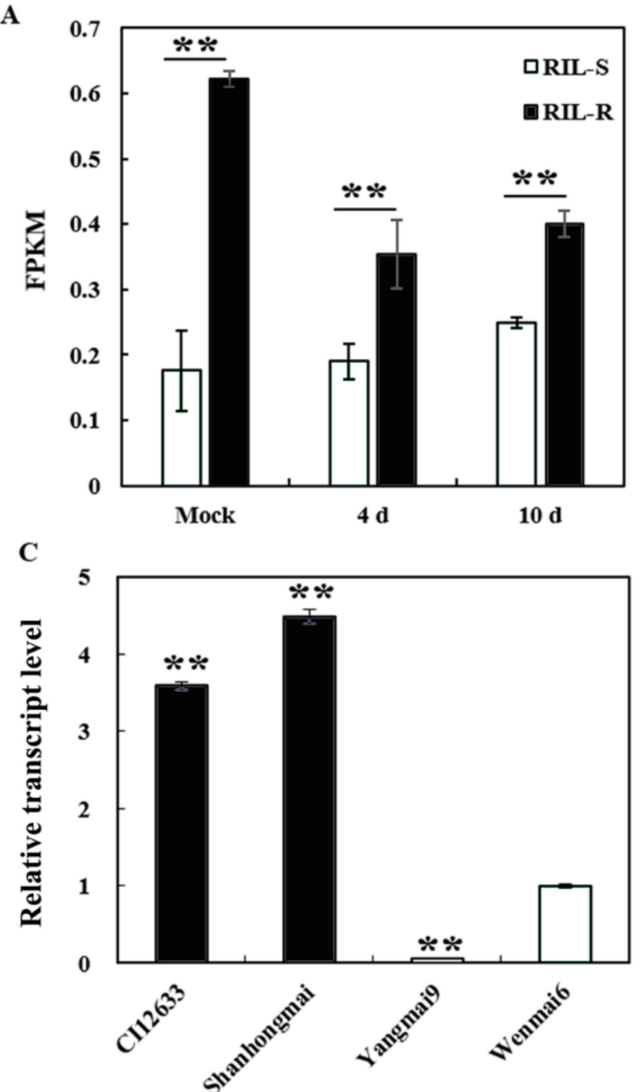

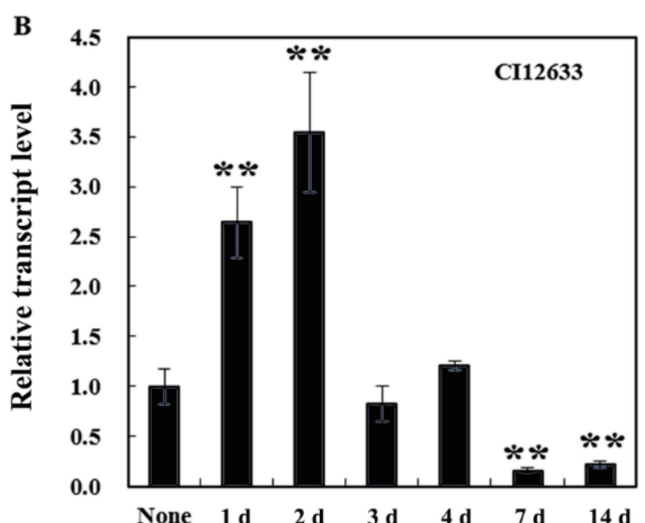

D

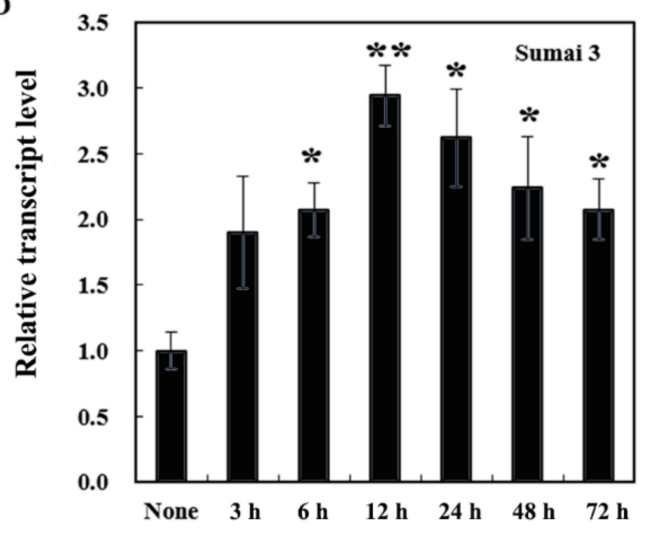

Figure 1. Transcriptional profiles of TaWAK2A-800 in the wheat responses to R. cerealis and F. graminearum. (A) RNA-sequencing data of TaWAK2A-800 transcription between RIL-R and RIL-S. (B) qRTPCR analysis of TaWAK2A-800 in the resistant wheat cultivar, CI12633, inoculated with the pathogen, R. cerealis. (C) qRT-PCR analysis of TaWAK2A-800 in four wheat cultivars with different degrees of sharp eyespot resistance at $2 \mathrm{dpi}$ with $R$. cerealis. The transcriptional level of TaWAK2A-800 in the Wenmai 6 was set to 1. (D) qRT-PCR analysis of TaWAK2A-800 in the resistant wheat cultivar, Sumai 3, plants inoculated with F. graminearum. The transcriptional level of TaWAK2A-800 without treatment (none) is set to 1 . Student's $t$-test $\left({ }^{*} p<0.05 ;{ }^{* *} p<0.01\right)$. Bars indicate standard error of the mean from three biological replications. The wheat actin gene was used as an internal control.

Moreover, we examined the relative transcript level of TaWAK2A-800 in the wheat response to the pathogen, $R$. cerealis, by qRT-PCR. The analyses showed that the TaWAK2A-800 gene transcript was rapidly induced in the sharp-eyespot-resistant wheat cultivar, CI12633, at day 1 , post inoculation (dpi) and $2 \mathrm{dpi}$, and reached a peak at $2 \mathrm{dpi}$ (Figure 1B). Interestingly, at $2 \mathrm{dpi}$, with $R$. cerealis compared with the sharp-eyespot-susceptible cultivars, Yangmai 9 and Wenmai 6, TaWAK2A-800 transcript abundance was significantly higher in the sharp-eyespot-resistant wheat cultivars (CI12633 and Shanhongmai) (Figure 1C). This suggests that the transcript abundance of TaWAK2A-800 might correspond to the resistance degree of the wheat cultivars. In addition, the transcript of TaWAK2A-800 was significantly induced upon $F$. graminearum inoculation in the FHB-resistant wheat cultivar, Sumai 3 (Figure 1D), similar to the expression patterns of two FHB-resistance genes studied in previous papers $[18,19]$. The above results suggests that TaWAK2A-800 was involved in the wheat defense responses, not only against $R$. cerealis, but also against $F$. graminearum.

\subsection{Sequence Characterization of TaWAK2A-800 in Wheat}

The TaWAK2A-800 genomic sequence was cloned from DNA taken from the stems of the wheat cultivar, Wenmai 6 . According to a BLAST analysis against the hexaploid wheat genome sequence, the full-length cDNA coding sequence of TaWAK2A-800 contains 
a complete open reading frame (ORF) with $2151 \mathrm{bp}$, and the gene locates on the short arm of wheat chromosome 2A. A comparison of the cDNA and genomic sequences indicates that the TaWAK2A-800 genomic sequence contains four exons and three introns (Figure 2A). The deduced protein, TaWAK2A-800, was comprised of 716 amino acid (aa) residues, its molecular weight was $77.88 \mathrm{kD}$, and the theoretical isoelectric point $(\mathrm{pI})$ was 6.65. There was a predicted signal peptide (at no. 1-18 amino acids, aa) in the N-terminal region, an EGF-like domain (no. 277-314 aa), and EGF-CA (no. 315-367 aa), with four conserved cysteine residues. Moreover, the C-terminal of the predicted TaWAK2A-800 harbored a cytosolic STK domain (no. 434-697 aa), which included an ATP binding site and an acting site (Figure 2B). All the structural characteristics of the predicted TaWAK2A-800 protein were coincident with those of the reported WAK proteins. Thus, TaWAK2A-800 might be a putative WAK protein. Because bread wheat is hexaploid and contains A, B, and $\mathrm{D}$ sets of genomes, three copies of TaWAK2A-800 were expected. In fact, after BLAST in the chromosome-based draft sequence of the hexaploid wheat using TaWAK2A-800, only one homoeologous gene from the wheat cultivar Chinese Spring was found on the wheat chromosome 2D, named as TaWAK-2D-600 (TraesCS2D02G070600.1), with a 2139 bp ORF. A pairwise comparison showed that the ORF and the amino acid sequences of TaWAK2A-800 shared $93.18 \%$ and $89.42 \%$ identities, respectively, with those of TaWAK-2D-600.

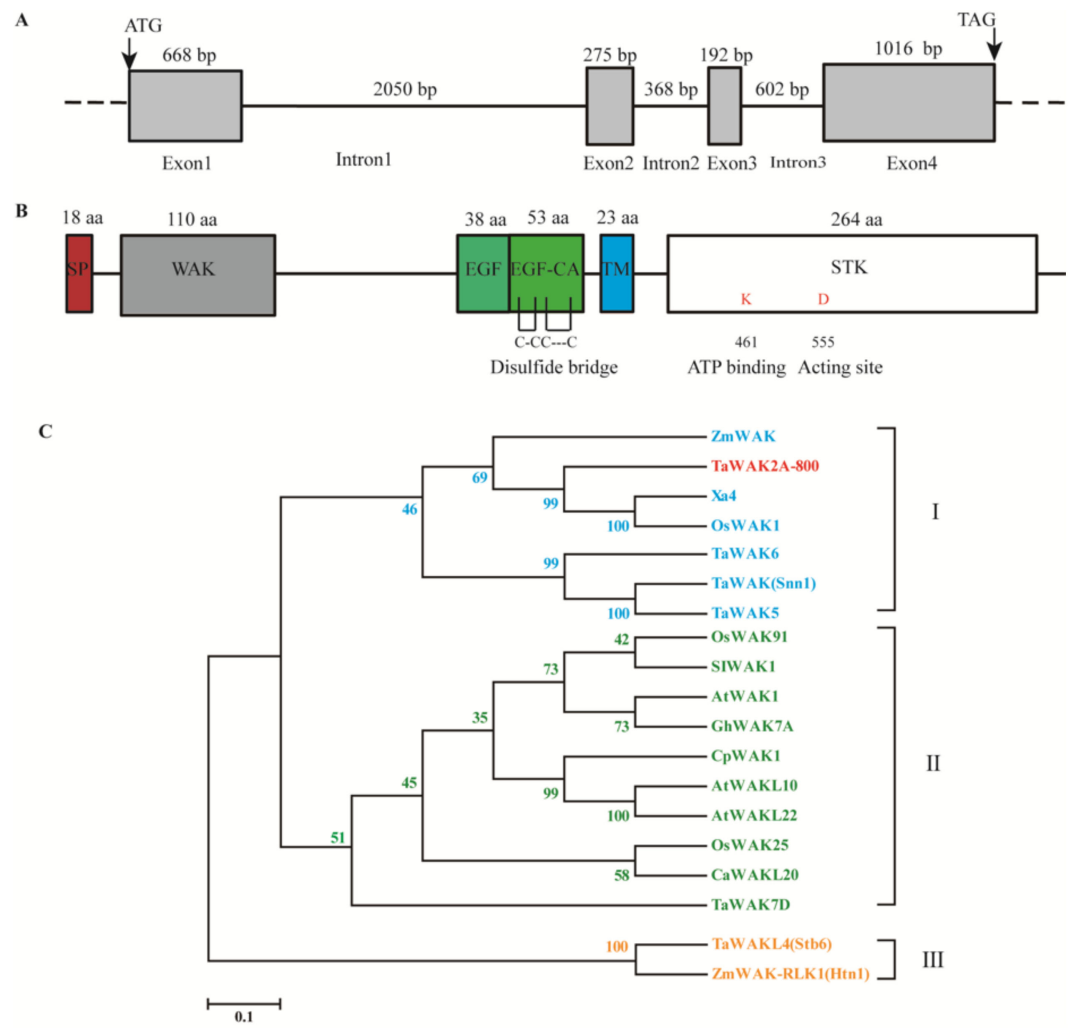

Figure 2. Sequence and phylogenetic analyses of TaWAK2A-800. (A) Genomic structure of the TaWAK2A-800 gene. (B) Schematic diagram of the domain (shaded area) of the TaWAK2A-800 protein. The red box represents a signal peptide, the gray box represents the WAK-GUB domain, the green boxes represent EGF domains, the blue box represents the transmembrane domain, and the white box represents the STK domain. (C) Phylogenetic analysis of TaWAK2A-800 protein and 18 other WAK/WAKL proteins. The bootstrapped phylogenetic tree is constructed by using the neighbor-joining phylogeny of MEGA 7.0.

Phylogenetic analysis was performed to decipher the relationship among TaWAK2A800 and the other 18 related WAKs/WAKLs from wheat, rice, maize, A. thaliana, cotton (Gossypium hirsutum), Craterostigma plantagineum, Capsicum annuum, and Solanum lycoper- 
sicum. The phylogenetic tree indicated that TaWAK2A-800 with Xa4 and OsWAK1 from rice, TaWAK5, TaWAK6, and TaWAK (Snn1) from wheat, and ZmWAK (encoded by qHSR1) from maize, were clustered on the same clade. Other WAKs were clustered into the second clade, including GhWAK7A from cotton, AtWAK1, AtWAKL10 and AtWAKL22 from A. thaliana, OsWAK91 and OsWAK25 from rice, CaWAKL20 from Capsicum annuum, CpWAK1 from $C$. plantagineum, TaWAK7D from wheat, and SIWAK1 from Solanum lycopersicum. Meanwhile, TaWAKL4 (Stb6) and ZmWAK-RLK (Htn1) were clustered into the third clade (Figure 2C). Moreover, we further compared the protein sequences of TaWAK2A-800 and five reported wheat WAKs to determine their identity. TaWAK2A-800 shared $35.63 \%, 35.52 \%, 31.08 \%$, $34.71 \%$, and $18.44 \%$ protein sequence identities with five wheat known-functional WAKs, namely, TaWAK5, TaWAK6, TaWAK7D, TaWAK (Snn1), and TaWAKL4 (Stb6), respectively. The above results indicate that TaWAK2A-800 is a WAK member that is distinct from the five reported wheat WAKs.

\subsection{Knocking-Down TaWAK2A-800 Compromises Wheat Resistance to Fusarium Head Blight and} Sharp Eyespot

To investigate whether the role of TaWAK2A-800 in wheat resistance to F. graminearum, TaWAK2A-800 was knocked-down (silenced) in the resistant wheat cultivar, Sumai 3, via the barley yellow dwarf virus (BSMV)-mediated VIGS method, and then the disease severities of these plants were assayed. Briefly, Si-Fi software prediction showed that the $172 \mathrm{bp}$ fragment specific to TaWAK2A-800 (Table 1) was screened to be a VIGS fragment, and then subcloned in the multiclone site of the BSMV $\gamma$ chain in order to generate the BSMV:TaWAK2A-800 recombinant construct.

Table 1. The VIGS fragment was analyzed by Si-Fi software.

\begin{tabular}{ccc}
\hline Target Genes & Total siRNA Hits & Efficient siRNA Hits \\
\hline TraesCS2A02G071800.1 & 71 & 35 \\
TraesCS2D02G070600.1 & 50 & 22 \\
\hline
\end{tabular}

After transfection with BSMV: TaWAK2A-800, or BSMV:GFP viruses, for 10 days, the leaves of the transinfected wheat Sumai 3 plants showed mild chlorotic mosaic symptom of BSMV (Figure 3A). RT-PCR analysis showed that the transcription of BSMV coat protein $(C P)$ in the wheat plants transfected by BSMV:GFP, or BSMV:TaWAK2A-800, at 15 days could be detected (Figure 3B), proving that these Sumai 3 plants were successfully infected by BSMV: TaWAK2A-800, or BSMV:GFP viruses. Further, the qRT-PCR analysis suggested that, compared with the control (BSMV: GFP transfected Sumai3) plants, the expression of TaWAK2A-800 was markedly downregulated in the BSMV: TaWAK2A-800-infected Sumai 3 plants (Figure 3C). To assess the resistance function of TaWAK2A-800 to FHB, the inoculum of the F. graminearum strain, F0609 (100 spores/ $\mu \mathrm{L})$, were injected into the spikelets of the spikes of the BSMV-transfected Sumai 3 using a syringe. At 20 dpi with F. graminearum, the FHB symptoms in the TaWAK2A-800-silenced Sumai 3 plants were more severe than in BSMV: GFP-transfected Sumai 3 (control) plants (Figure 3D). Accordingly, in two batches, the average number of scabbed spikelets, and the average percentage of the scabbed spikelets of the TaWAK2A-800-silenced Sumai 3 plants, were 3.85 and 4.36, $36.99 \%$ and $39.87 \%$, respectively. Meanwhile, the average number of scabbed spikelets, and the average percentage of the scabbed spikelets of BSMV:GFP-treated Sumai 3 plants, were 2.76 and $3.18,26.67 \%$ and $28.23 \%$, respectively. The results suggest that the knockingdown of TaWAK2A-800 in Sumai 3 compromised the resistance of the wheat cv. Sumai 3 to FHB caused by F. graminearum, suggesting that TaWAK2A-800 expression is required for the resistance of the $F$. graminearum-resistant wheat cv. Sumai 3 to FHB caused by F. graminearum. 


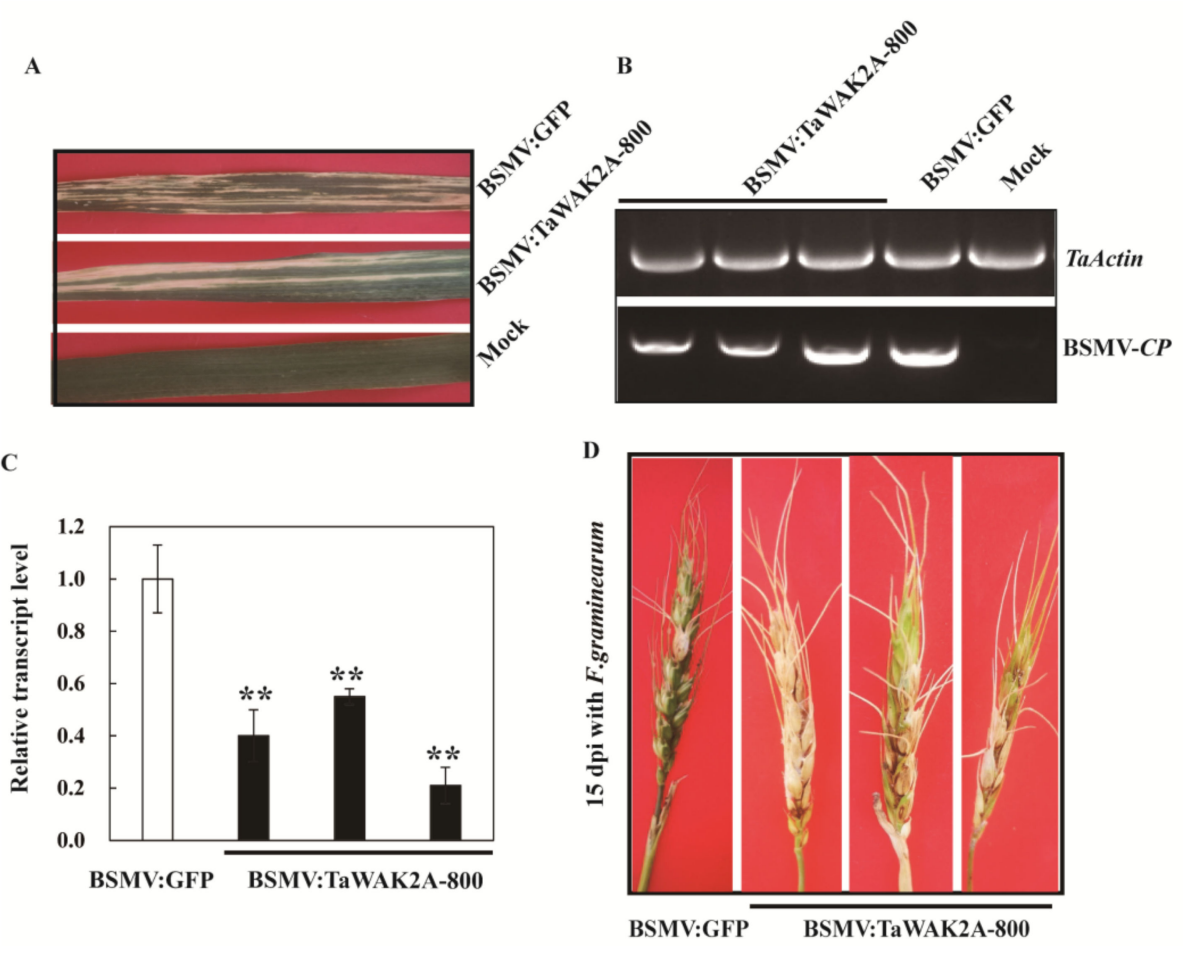

Figure 3. Silencing of TaWAK2A-800 by BSMV-induced gene silencing increases Sumai 3 susceptibility to F. graminearum. (A) Mild chlorotic mosaic symptom displayed on the newly emerged leaves at 15 dpi with BSMV:GFP or BSMV:TaWAK2A-800. (B) RT-PCR analysis of the transcription of BSMV $C P$ in the wheat plants transfected by BSMV:GFP or BSMV:TaWAK2A-800 at 15 days. (C) qRT-PCR analysis of the TaWAK2A-800 transcript abundance in the Sumai 3 wheat plants transfected by BSMV:GFP or BSMV:TaWAK2A-800 at 15 dpi. The transcript level of TaWAK2A-800 in BSMV:GFPtransfected Sumai 3 is set to 1 . Student's $t$-test $\left.{ }^{* *} p<0.01\right)$. Bars indicate standard error of the mean from three biological replications. Three replicates were averaged and statistically treated ( $t$-test: $\left.{ }^{* *} p<0.01\right)$. Bars indicated standard error of the mean. (D) FHB symptoms of the control and TaWAK2A-800-silenced Sumai 3 spikes at 20 dpi with F. graminearum.

To examine whether TaWAK2A-800 expression is also required for the innate immunity of the wheat plant to $R$. cerealis, TaWAK2A-800 transcript was knocked-down by VIGS in the $R$. cerealis-resistant wheat $\mathrm{cv}$. CI12633. Fifteen days post the virus transfection into the wheat leaves, the mild chlorotic mosaic symptoms and CP of the BSMV transfection could be observed on newly emerged leaves (Figure 4A) and was readily detected in the virus-transfected leaves, but not in the mock plants (Figure 4B), indicating that the BSMV-treated wheat plants were successfully transfected by BSMV. Compared with BSMV: GFP-transfected CI12633 plants, the TaWAK2A-800 transcription was substantially knocked-down/silenced in BSMV:TaWAK2A-800-transfected CI12633 plants (Figure 4C). Furthermore, the BSMV: GFP-transfected, and the BSMV: TaWAK2A-800-silenced, CI12633 plants were inoculated with the pathogenic R. cerealis strain, WK207. Microscopic observation showed that, at $7 \mathrm{dpi}$ with WK207, the R. cerealis hyphae density on the inoculated base sheaths of the TaWAK2A-800-silenced wheat plants was more than that on the BSMV:GFPtransfected wheat plants (Figure 4D), providing evidence that silencing TaWAK2A-800 impaired the resistance of CI12633 against the hyphae development of $R$. cerealis. At $15 \mathrm{dpi}$, with the pathogen $R$. cerealis, brown lesions (the typical symptoms of sharp eyespot) displayed on the sheaths and stems of the TaWAK2A-800-silenced CI12633 plants but displayed, to a lesser extent, on the sheaths and stems of the BSMV: GFP-treated (control) CI12633 (Figure 4E). Moreover, with the pathogen R. cerealis, at $35 \mathrm{dpi}$, the stems of the TaWAK2A-800-silenced CI12633 plants presented more serious necrotic areas than the BSMV: GFP-infected CI12633 plants (Figure 4E). In two batches of VIGS and disease scor- 
ing at 35 dpi with the pathogen, R. cerealis WK207, the average infection types (ITs) on the stems of the TaWAK2A-800-silenced wheat cultivar CI12633 plants were between 2.50 and 2.89 (Figure 4F), while those of the BSMV:GFP-infected CI12633 (control) plants were 1.71 to 1.93 , respectively. These results indicate that knocking-down TaWAK2A-800 in the wheat cultivar, CI12633, could cause higher susceptibility to the pathogen $R$. cerealis, and suggests that TaWAK2A-800 was required for wheat-resistance responses against pathogenic $R$. cerealis infection.

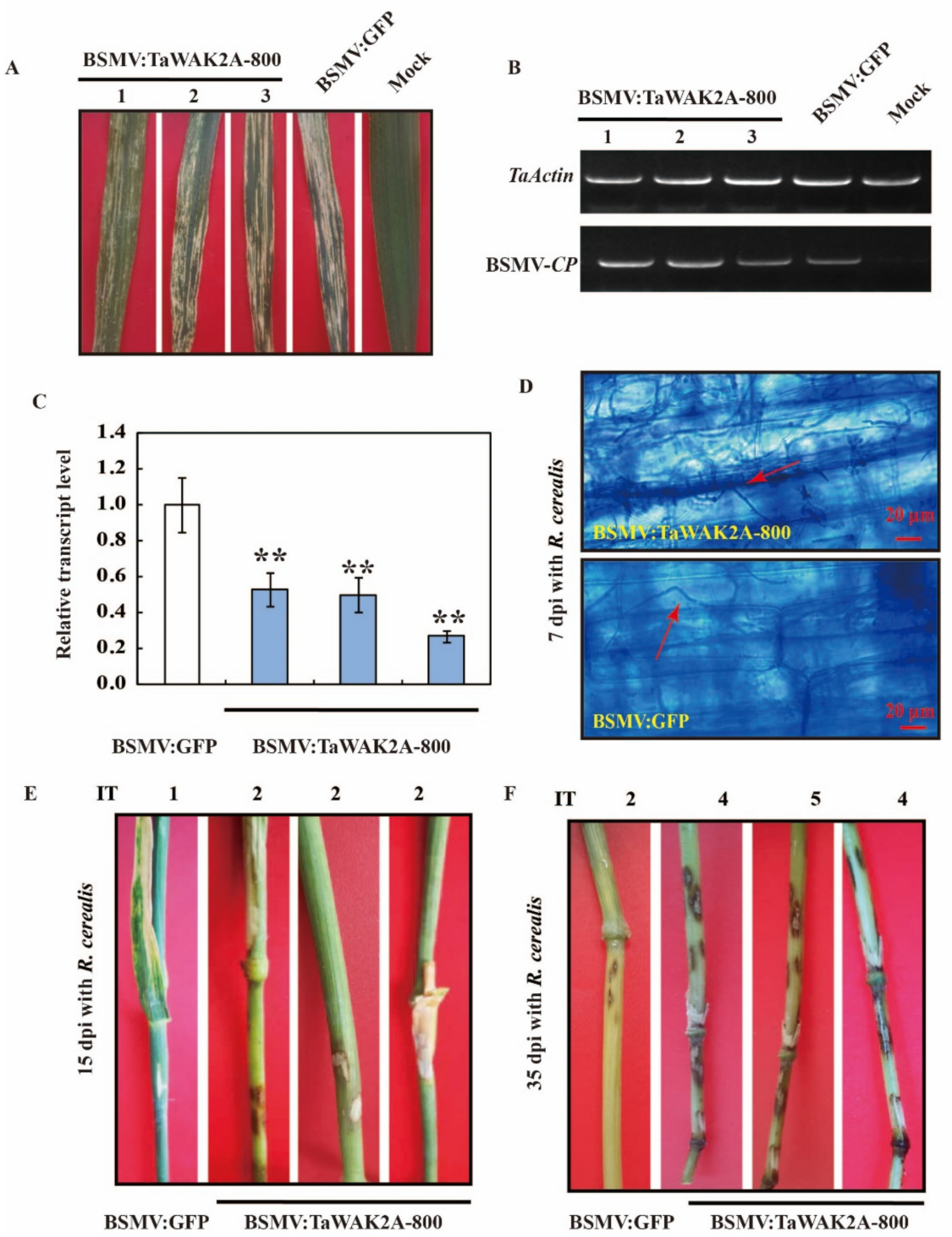

Figure 4. Knocking-down of TaWAK2A-800 by BSMV-induced gene silencing impairs resistance of the wheat cv. CI12633 to the pathogen R. cerealis. (A) Mild chlorotic mosaic symptoms displayed on newly emerged leaves at 15 dpi with BSMV:GFP, or BSMV: TaWAK2A-800, transfection. (B) RTPCR analysis of the transcription of BSMV CP in the wheat plants transfected by BSMV:GFP, or BSMV:TaWAK2A-800, for $15 \mathrm{~d}$. (C) qRT-PCR analysis of the transcript abundance of TaWAK2A-800 in the CI12633 wheat plants transfected by BSMV:GFP, or BSMV:TaWAK2A-800, for $15 \mathrm{~d}$. The transcript level of TaWAK2A-800 in BSMV:GFP-transfected CI12633 is set to 1 . Student's $t$-test $\left({ }^{* *} p<0.01\right)$. (D) The R. cerealis hyphae on the base leaf sheath of the BSMV: TaWAK2A-800- and BSMV:GFPtransfected CI12633 plants at $7 \mathrm{dpi}$ with $R$. cerealis, WK207, were stained with trypan blue. Bar $=20 \mu \mathrm{m}$. (E) Wheat sharp eyespot symptoms of BSMV:GFP- and BSMV:TaWAK2A-800-transfected CI12633 stems at 15 dpi with $R$. cerealis. (F) Wheat sharp eyespot symptoms on BSMV:GFP-transfected and BSMV:TaWAK2A-800-silenced CI12633 stems at 35 dpi with R. cerealis WK207. 


\subsection{TaWAK2A-800 May Contribute to Chitin-Induced Defense Pathway}

As a conserved component of the fungal cell wall, chitin can trigger plant immune response [20]. To investigate how TaWAK2A-800 responds to exogenous chitin stimuli, we analyzed the transcript profiles of TaWAK2A-800 in wheat cv. CI12633 treated with $100 \mu \mathrm{g} / \mathrm{mL}$ chitin [21], or with mock solution, for 5, 10, 20, and $30 \mathrm{~min}$. The analyses showed that the TaWAK2A-800 transcript level was significantly elevated by chitin treatment compared with the mock treatment (Figure 5).

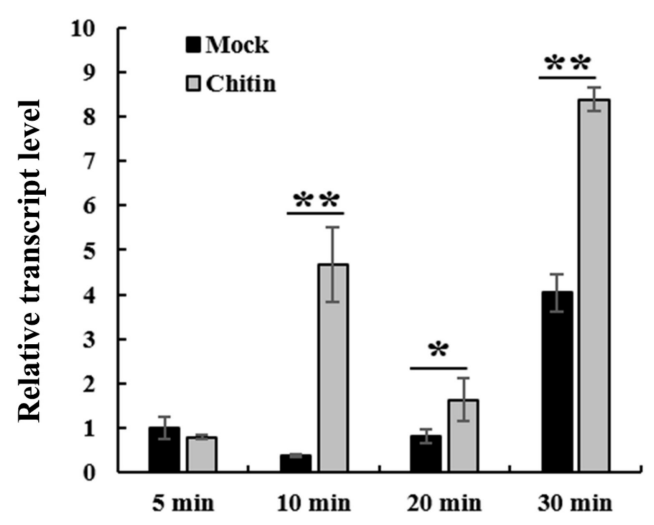

Figure 5. TaWAK2A-800 contributed to chitin-induced immune pathway. Expression patterns of TaWAK2A-800 in wheat cultivar CI12633 plants after treatment with exogenous chitin for 5, 10, 20, and $30 \mathrm{~min}$. Student's $t$-test $\left({ }^{*} p<0.05 ;{ }^{* *} p<0.01\right)$. Bars indicate standard error of the mean from three biological replications. TaActin was used as an internal control.

\subsection{Silencing TaWAK2A-800 Decreases the Transcripts of Defense-Related Genes in Wheat}

To investigate if the silencing of TaWAK2A-800 represses the expression of defenseassociated genes in wheat, qRT-PCR was deployed to examine the transcription levels of wheat defense-associated genes in TaWAK2A-800-silenced and BSMV:GFP-transfected wheat plants inoculated with the pathogens $R$. cerealis, or F. graminearum. As is shown in Figure $6 \mathrm{~A}, \mathrm{~B}$, after inoculation with $R$. cerealis and F. graminearum, the expression levels of the wheat receptor-like kinase-coding gene, TaCERK1, the wheat receptor-like cytoplasmic kinase, TaRLCK1B, and the wheat mitogen-activated protein kinase, TaMPK3, were decreased in the BSMV:TaWAK2A-800-infected wheat plants compared with the BSMV:GFP-transfected wheat plants. Moreover, compared with the BSMV:GFP-transfected (control) wheat plants, the transcript levels of three wheat defense-marker genes, including defensin, chitinase3, and $\beta-1,3-$ glucanase, were significantly reduced in TaWAK2A-800silenced wheat plants after $R$. cerealis inoculation (Figure $6 \mathrm{~A}$ ). In addition, after inoculation with $F$. graminearum, only $\beta-1,3-$ Glucanase expression was significantly downregulated in TaWAK2A-800-silenced Sumai 3 wheat plants compared with the BSMV:GFP-infected (control) plants (Figure 6B). These data suggest that expressed TaWAK2A-800 is also required for the expression of these defense-associated genes. 


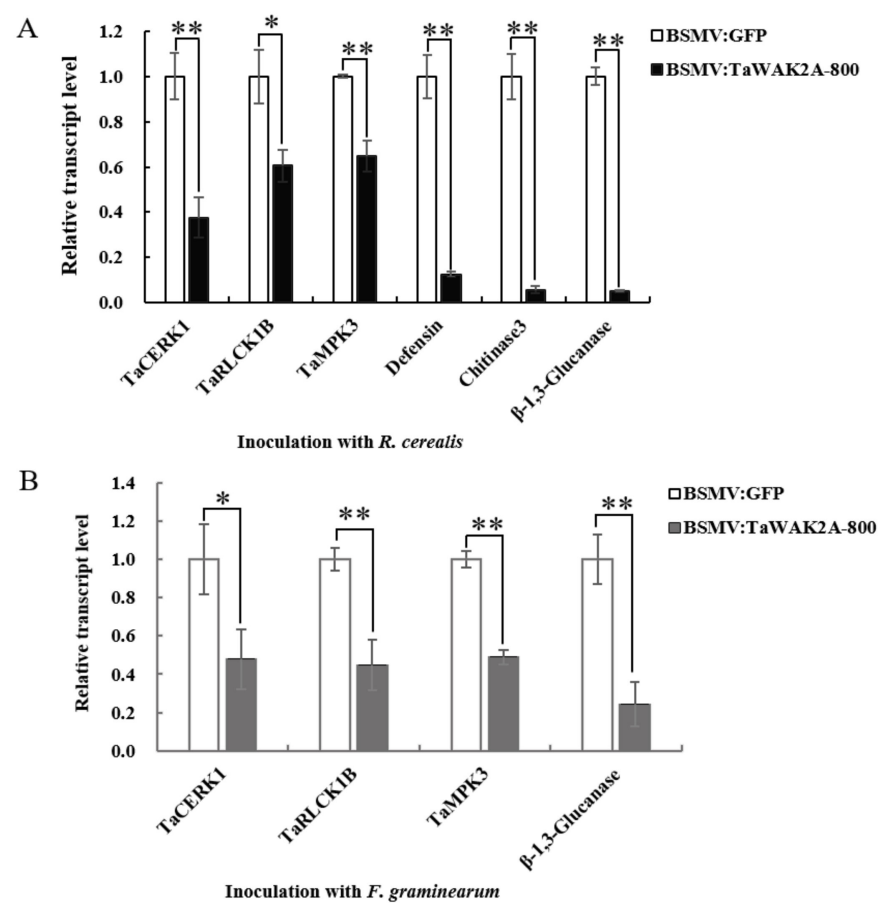

Figure 6. Transcription profiles of defense-associated genes TaCERK1, TaRLCK1B, TaMPK3, Defensin, Chitinase3, and $\beta-1,3-$ Glucanase in BSMV: GFP-transfected, and BSMV:TaWAK2A-800-silenced, wheat plants inoculated with (A) R. cerealis and (B) F. graminearum. Statistically significant differences between BSMV: GFP-transfected and BSMV:TaWAK2A-800-silenced wheat plants were determined using Student's $t$-test $\left({ }^{*} p<0.05 ;{ }^{* *} p<0.01\right)$. Bars indicate standard error of the mean from three biological replications. TaActin was used as an internal control.

\section{Discussion}

In Arabidopsis, rice, maize, cotton, and wheat, some WAK/WAKL proteins were reported to have an important function in resistance responses to the bacterial or fungal pathogens [14-17,21-26]. In this study, on the basis of the RNA-seq data and qRT-PCR analyses, we identified the wheat-wall-associated kinase gene, TaWAK2A-800, with broadspectrum resistance against $R$. cerealis and F. graminearum. TaWAK2A-800 belongs to the WAK subfamily, but its protein sequence is distinct from those of the five reported wheat WAKs, including TaWAK5, TaWAK6, TaWAK7D, TaWAK (Snn1), and TaWAKL4 (Stb6). The deduced protein sequence of TaWAK2A-800 contains one signal peptide, one galacturonanbinding GUB domain, one EGF-like domain, one EGF-CA domain, one transmembrane region, and a non-RD kinase domain. Among various types of RLKs, non-RD RLKs predominantly bind to native immune receptors, recognize conserved microbial characteristics, activate PTI, and even confer disease resistance [27]. For instance, four reported WAK proteins, including ZmWAK-RLK1 [13], ZmWAK [14], Xa4 [15], and TaWAK6 [17], all contain a non-RD kinase domain that mediates disease resistance. Our functional analyses revealed that TaWAK2A-800 also belongs to a non-RD-type WAK protein in wheat, and positively participates in the resistance responses of wheat to sharp eyespot and FHB.

Further, qRT-PCR analyses showed that, compared with nontreatment, the expression level of TaWAK2A-800 in wheat was significantly upregulated by F. graminearum infection, or in the early infection stage (1-2d), of the pathogen $R$. cerealis. Intriguingly, after inoculation with $R$. cerealis for $2 \mathrm{~d}$, the transcript level of TaWAK2A-800 was significantly higher in $R$. cerealis-resistant wheat cultivars, Shanhongmai and CI12633, than in the susceptible wheat cultivars, Yangmai 9 and Wenmai 6. Importantly, knocking-down TaWAK2A-800 impaired the resistance of wheat to both sharp eyespot caused by R. cerealis infection, and FHB caused by F. graminearum infection. A previous study reported that TaWAK2A-800 was responsible for FHB-resistance QTL in wheat [18]. Herein, our functional dissection results 
were not only in line with the aforementioned reported conclusion, but also revealed a new defensive role for TaWAK2A-800 in the wheat immune response to sharp eyespot. Further, the current study reveals a potentially conserved mechanism of wheat resistance against F. graminearum and $R$. cerealis.

Previous studies have shown that three types of $P R$-encoding genes, including $D e$ fensin, Chitinases, and $\beta-1,3-$ Glucanase, contribute to the resistance of wheat to sharp eyespot $[28,29]$. To understand the potential molecular mechanisms of TaWAK2A-800 in the defense response against both pathogens, we examined the transcripts of Defensin, Chitinase 3, and $\beta-1,3-$ Glucanase in BSMV: TaWAK2A-800-silenced wheat, and the BSMV:GFPtransfected wheat plants, inoculated with $R$. cerealis or F. graminearum. The results show that, compared with BSMV:GFP-transfected wheat plants, the transcript levels of the wheat, Defensin, Chitinase 3, and B-1,3-Glucanase were lower in the more susceptible TaWAK2A-800silenced wheat plants after inoculation with $R$. cerealis or F. graminearum. The data suggest that TaWAK2A-800 might indirectly activate the expression of the above defense-marker genes in wheat resistance responses against $R$. cerealis or F. graminearum.

Chitin, a typical pathogen-associated molecular pattern (PAMP) on the fungal cell wall, can trigger the plant innate immune response by activating PAMP-triggered immunity (PTI) in many plant species [30]. In Arabidopsis and rice, the receptor complex, composed of LYK5 and CERK1 (an RLK with an extracellular lysine motif domain), can recognize fungal chitin [31-33]. Upon chitin perception, CERK1 phosphorylates the RLCK PBL27 in Arabidopsis [34]. In the knockout mutation of PBL27, the chitin-dependent activation of the MAPKs (MPK3 and MPK6) was markedly reduced [35]. In cotton, the wall-associated kinase, GhWAK7A, interacts with the chitin sensory receptors, GhCERK1 and GhLYK5, to phosphorylate GhLYK5, activating the chitin-induced defense responses to Verticillium dahliae and Fusarium oxysporum f.sp. vasinfectum [21]. In this study, the TaWAK2A-800 transcript level was significantly induced by chitin treatment. Moreover, compared to the control wheat plants, the transcript abundance of the chitin-induced immune pathway marker genes, TaCERK1, TaRLCK1B, and TaMPK3, were reduced in TaWAK2A-800-silenced wheat plants after inoculation with $R$. cerealis and $F$. graminearum. These results suggest that TaWAK2A-800 contributes to the wheat defense responses to $R$. cerealis and F. graminearum, possibly through a chitin-induced defense pathway. Similarly, the transcripts of rice OsWAK90/91 were upregulated after chitin treatment, and boosted resistance against rice blast caused by Magnaporthe oryzae [22].

\section{Materials and Methods}

\subsection{Plants Material, Fungal Isolates, and Primers}

Four wheat cultivars/varieties, including R. cerealis-resistant CI12633 (Prof. Shibing Cai, Jiangsu Academy of Agricultural Sciences, Nanjing, Jiangsu, China), and Shanhongmai (Prof. Jizeng Jia, Institute of Crop Sciences, CAAS, Beijing, China), R. cerealis-susceptible Yangmai 9 (Prof. Jizeng Jia), and Wenmai 6 (Prof. Shunhe Cheng, Jiangsu Academy of Agricultural Sciences, Nanjing, Jiangsu, China) [36], were used to investigate the transcriptional profile of TaWAK2A-800. The sharp-eyespot-resistant and -susceptible recombinant RILs (derived from Shanhongmai and Wenmai 6 cross) were used for RNA-sequencing, and RNA-sequencing analyses were performed as described by Guo et al. [37]. The R. cerealis-resistant cultivar, CI12633, and the F. graminearum-resistant cultivar, Sumai 3 (Prof. Jizhong wu, Jiangsu Academy of Agricultural Sciences, Nanjing, Jiangsu, China), were used to detect the defense role of TaWAK2A-800 against $R$. cerealis and F. graminearum by the virus-induced gene-silencing (VIGS) experiment, respectively. The fungal pathogenic R. cerealis isolate, WK207, and the F. graminearum isolate, F0609, were provided by Prof. Jinfeng Yu (Shandong Agricultural University, Tai'an, Shandong, China) and Prof. Miaoping Zhou (Jiangsu Academy of Agricultural Sciences, Nanjing, Jiangsu, China), respectively.

All wheat plants were grown in a glasshouse in 14 -h light $/ 10-\mathrm{h}$ dark $\left(22^{\circ} \mathrm{C} / 12{ }^{\circ} \mathrm{C}\right)$ conditions. At the tillering growth stage, these wheat plants (CI12633, Shanhongmai, Yangmai 9, Wenmai 6) were further inoculated on each stem base with small toothpicks 
harboring the well-developed mycelia of $R$. cerealis. Then, the wheat plants were grown in a growth chamber at $22{ }^{\circ} \mathrm{C}$ and high humidity during the first 14 days. To prepare conidial suspensions for inoculation, F. graminearum mycelia were collected from potato agar plates, then transferred into liquid mung bean medium, and cultivated at $25^{\circ} \mathrm{C}$ for $3 \mathrm{~d}$ with shaking at $150 \mathrm{rpm}$. The conidial suspension was filtered, centrifuged, resuspended, and adjusted to $100 / \mu \mathrm{L}$ in sterile water. At the anthesis of Sumai 3, the suspension was further injected into a spikelet of spike using a syringe. Then, these spikes of Sumai 3 were placed in a plastic moist chamber, with high humidity, at $22{ }^{\circ} \mathrm{C}$ for 2 days. The RNAsequencing-based transcriptomics of TaWAK2A-800 (Table S1) were subjected as described by Guo et al. [37].

Chitin was suspended in sterile water and used at a final concentration of $100 \mu \mathrm{g} / \mathrm{mL}$ [24]. Wheat seedlings were sprayed with distilled water (mock), or chitin solution. At 5, 10, 20, and $30 \mathrm{~min}$, we collected the wheat leaves corresponding to each treatment, and extracted RNAs for RT-qPCR analysis.

\subsection{Cloning and Sequence Analysis of TaWAK2A-800}

Total plant RNA extraction was performed following Zhang et al. [38]. This TaWAK2A800 gene sequence was cloned from the wheat cultivar Wenmai 6 leaves based on the means of two rounds of nest PCR reactions by gene-specific primer (TaWAK2A-800-F1: 5' CAACGCCACACTATCCAGGT-3', TaWAK2A-800-R1: 5' -TGTTCCC TTCCCACCTCTAGT3'; TaWAK2A-800-F2: 5'-CATCAGGTCGACACAGCAGG-3', TaWAK2A-800-R2: 5'-CAGCC GGGAGATTAGGAAGC-3') following the above sequence. TraesCS2A02G071800.1 was downloaded from the Chinese Spring V1.0 genome (http:/ /202.194.139.32/jbrowse.html, accessed on 27 February 2020). The deduced protein sequence of TaWAK2A-800 and the phylogenetic tree were obtained and constructed as described by Guo et al. [37].

\subsection{Assay for the Function of TaWAK2A-800 in Wheat Defense against FHB and Sharp Eyespot}

The VIGS fragment (172 bp) of TaWAK2A-800, and its specificity and efficient siRNA hits, were predicted by the Si-Fi software (http:/ / labtools.ipk-gatersleben.de/, accessed on 24 April 2020). The specific fragment of TaWAK2A-800 was amplified from the cDNA sequence for TaWAK2A-800 using a gene-specific primer (TaWAK2A-800- $\gamma$-F: $5^{\prime}$-CTAGCTAGC GCCTACCATGGGAGAAGTCAG-3', TaWAK2A-800- $\gamma$-R: 5'-CTAGCTAGCACATCACACA CTGGAGAAAACA-3') from the CI12633, and inserted in an antisense orientation into the site of the RNA $\gamma$ of the part of the BSMV to generate a BSMV: TaWAK2A-800 construct. Following Zhu et al. [36], the mixtures of the transcribed RNAs of the tripartite DNA chains of BSMV: TaWAK2A-800, and BSMV: GFP (as control), were separately transfected. At least 20 CI12633 or Sumai 3 wheat plants were transfected at the three-leaf stage, and then moisturized for at least $2 \mathrm{~d}$. The wheat tissue samples were harvested and analyzed to monitor BSMV infection at $15 \mathrm{~d}$ after virus inoculation by a gene-specific primer (BSMV-CP-F: $5^{\prime}-$ TGACTGCTAAGGGTGGAGGA-3', BSMV-CP-R: 5'-CGGTTGAACATCACGAA GAGT-3'). Following the previous method [29,39], the BSMV-transfected Sumai 3 or CI12633plants were inoculated with the F. graminearum isolate, F0609, or the R. cerealis isolate, WK207, at 15 days transfected with BSMV, and then scored for disease symptoms at 20 and 35 days post the fungal inoculation.

\section{4. $R T-P C R$ and $q R T-P C R$}

The transcriptional levels of TaWAK2A-800 (TaWAK2A-800-QF: 5'-GGTATCGTGCTAC TGGAGCTC-3', TaWAK2A-800-QR: 5'-CTCCCATGGTAGGC CTGTTA-3'), TaRLCK1B [40], TaCERK1 (TaCERK1-QF: 5'-CGGAGCAGATGGAC ACACTT-3', TaCERK1-QR: 5'-TGAGTG

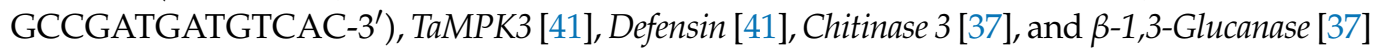
in wheat were measured and analyzed using RT-PCR or qRT-PCR. Following the previous method [37], the qRT-PCR reaction was performed by using a SYBR Premix Ex Taq kit (TaKaRa, Otsu, Japan), in volumes of $20 \mu \mathrm{L}$, on an ABI 7500 RT-PCR system (Applied Biosystems, Waltham, MA, USA). The $2^{-\Delta \Delta \mathrm{CT}}$ method [42] was used to calculate the relative 
transcription level of the target genes (internal reference: TaActin). Three independent replications were performed in each test.

\section{Conclusions}

TaWAK2A-800 was expressed to a higher extent in the $R$. cerealis-resistant wheat accessions than in the susceptible wheat ones. The gene transcript abundance is induced after F. graminearum infection and chitin treatment. The expressed TaWAK2A-800 is required for the resistance of wheat to $R$. cerealis and F. graminearum by promoting the expression of TaCERK1, TaRLCK1B, TaMPK3, Defensin, Chitinase 3, and $\beta$-1,3-Glucanase. This work reveals a novel role for the wheat TaWAK2A-800 gene in wheat plant immunity against pathogens. TaWAK $2 A-800$ is a potentially important gene for improving the broad-spectrum resistance of wheat to $R$. cerealis and F. graminearum.

Supplementary Materials: The following are available online at https:/ / www.mdpi.com/article/10 $.3390 /$ ijms222111493/s1.

Author Contributions: Z.Z. designed the research, was the program funding winner, and wrote and edited the manuscript; F.G. and T.W. performed the majority of the experiments; H.Q. performed the VIGS transfections; X.Z. extracted RNAs of RILs and kept RNA-seq data; and F.G. and G.X. wrote the Methods and Results in the draft manuscript. All authors have read and agreed to the published version of the manuscript.

Funding: This study was funded by the Chinese NSFC program (31771789).

Institutional Review Board Statement: Not applicable.

Informed Consent Statement: Not applicable.

Data Availability Statement: The data presented in this study are available on request from the corresponding author.

Acknowledgments: All authors are very grateful to Jizeng Jia (Institute of Crop Sciences, CAAS) for providing the RIL population, Miaoping Zhou (Jiangsu Academy of Agricultural Sciences, China) for providing the F. graminearum isolate, F0609, and Zhenqi Su (Chinese Agricultural University, Beijing) for helping in the FHB assessment.

Conflicts of Interest: The authors declare no conflict of interest.

\section{References}

1. Savary, S.; Willocquet, L.; Pethybridge, S.J.; Esker, P.; McRoberts, N.; Nelson, A. The global burden of pathogens and pests on major food crops. Nat. Ecol. Evol. 2019, 3, 430-439. [CrossRef] [PubMed]

2. Wang, H.; Sun, S.; Ge, W.; Zhao, L.; Hou, B.; Wang, K.; Lyu, Z.; Chen, L.; Xu, S.; Guo, J.; et al. Horizontal gene transfer of Fhb7 from fungus underlies Fusarium head blight resistance in wheat. Science 2020, 368, 596. [CrossRef]

3. He, Y.; Wu, L.; Liu, X.; Jiang, P.; Yu, L.; Qiu, J.; Wang, G.; Zhang, X.; Ma, H. TaUGT6, a Novel UDP-Glycosyltransferase Gene Enhances the Resistance to FHB and DON Accumulation in Wheat. Front. Plant Sci. 2020, 11, 574775. [CrossRef]

4. Cheng, S.; Yao, J.; Zhang, X.; Ma, H. Breeding for the resistance to Fusarium head blight of wheat in China. Front. Agric. Sci. Eng. 2019, 6, 15. [CrossRef]

5. Hamada, M.S.; Yin, Y.; Chen, H.; Ma, Z. The escalating threat of Rhizoctonia cerealis, the causal agent of sharp eyespot in wheat. Pest Manag. Sci. 2011, 67, 1411-1419. [CrossRef]

6. Chen, L.; Zhang, Z.; Liang, H.; Liu, H.; Du, L.; Xu, H.; Xin, Z. Overexpression of TiERF1 enhances resistance to sharp eyespot in transgenic wheat. J. Exp. Bot. 2008, 59, 4195-4204. [CrossRef]

7. Hao, Y.; Rasheed, A.; Zhu, Z.; Wulff, B.B.H.; He, Z. Harnessing Wheat Fhb1 for Fusarium Resistance. Trends Plant Sci. 2020, $25,1-3$. [CrossRef]

8. Chen, J.; Li, G.H.; Du, Z.Y.; Quan, W.; Zhang, H.Y.; Che, M.Z.; Wang, Z.; Zhang, Z.J. Mapping of QTL conferring resistance to sharp eyespot (Rhizoctonia cerealis) in bread wheat at the adult plant growth stage. Theor. Appl. Genet. 2013, 126, $2865-2878$. [CrossRef]

9. Shiu, S.H.; Bleecker, A.B. Plant receptor-like kinase gene family: Diversity, function, and signaling. Sci. STKE 2001, 2001 , re22. [CrossRef] [PubMed]

10. Verica, J.A.; He, Z.H. The cell wall-associated kinase (WAK) and WAK-like kinase gene family. Plant Physiol. 2002, 129, 455-459. [CrossRef] [PubMed] 
11. Jo, Y.; Cho, W.K.; Rim, Y.; Moon, J.; Chen, X.Y.; Chu, H.; Kim, C.Y.; Park, Z.Y.; Lucas, W.J.; Kim, J.Y. Plasmodesmal receptor-like kinases identified through analysis of rice cell wall extracted proteins. Protoplasma 2011, 248, 191-203. [CrossRef] [PubMed]

12. Diener, A.C.; Ausubel, F.M. Resistance to fusarium oxysporum 1, a dominant Arabidopsis disease-resistance gene, is not race specific. Genetics 2005, 171, 305-321. [CrossRef]

13. Hurni, S.; Scheuermann, D.; Krattinger, S.G.; Kessel, B.; Wicker, T.; Herren, G.; Fitze, M.N.; Breen, J.; Presterl, T.; Ouzunova, M.; et al. The maize disease resistance gene Htn1 against northern corn leaf blight encodes a wall-associated receptor-like kinase. Proc. Natl. Acad. Sci. USA 2015, 112, 8780-8785. [CrossRef] [PubMed]

14. Zuo, W.; Chao, Q.; Zhang, N.; Ye, J.; Tan, G.; Li, B.; Xing, Y.; Zhang, B.; Liu, H.; Fengler, K.A.; et al. A maize wall-associated kinase confers quantitative resistance to head smut. Nat. Genet. 2015, 47, 151-157. [CrossRef]

15. Hu, K.; Cao, J.; Zhang, J.; Xia, F.; Ke, Y.; Zhang, H.; Xie, W.; Liu, H.; Cui, Y.; Cao, Y.; et al. Improvement of multiple agronomic traits by a disease resistance gene via cell wall reinforcement. Nat. Plants 2017, 3, 17009. [CrossRef]

16. Saintenac, C.; Lee, W.S.; Cambon, F.; Rudd, J.J.; King, R.C.; Marande, W.; Powers, S.J.; Bergès, H.; Phillips, A.L.; Uauy, C.; et al. Wheat receptor-kinase-like protein Stb6 controls gene-for-gene resistance to fungal pathogen Zymoseptoria tritici. Nat. Genet. 2018, 50, 368-374. [CrossRef]

17. Dmochowska-Boguta, M.; Kloc, Y.; Zielezinski, A.; Werecki, P.; Nadolska-Orczyk, A.; Karlowski, W.M.; Orczyk, W. TaWAK6 encoding wall-associated kinase is involved in wheat resistance to leaf rust similar to adult plant resistance. PLOS ONE 2020, 15, e0227713. [CrossRef] [PubMed]

18. Gadaleta, A.; Colasuonno, P.; Giove, S.L.; Blanco, A.; Giancaspro, A. Map-based cloning of QFhb.mgb-2A identifies a WAK2 gene responsible for Fusarium Head Blight resistance in wheat. Sci. Rep. 2019, 9, 6929. [CrossRef]

19. Cuthbert, P.A.; Somers, D.J.; Thomas, J.; Cloutier, S.; Brulé-Babel, A. Fine mapping Fhb1, a major gene controlling fusarium head blight resistance in bread wheat (Triticum aestivum L.). Theor. Appl. Genet. 2006, 112, 1465-1472. [CrossRef]

20. Shinya, T.; Nakagawa, T.; Kaku, H.; Shibuya, N. Chitin-mediated plant-fungal interactions: Catching, hiding and handshaking. Curr. Opin. Plant Biol. 2015, 26, 64-71. [CrossRef]

21. Wang, P.; Zhou, L.; Jamieson, P.; Zhang, L.; Zhao, Z.; Babilonia, K.; Shao, W.; Wu, L.; Mustafa, R.; Amin, I.; et al. The Cotton Wall-Associated Kinase GhWAK7A Mediates Responses to Fungal Wilt Pathogens by Complexing with the Chitin Sensory Receptors. Plant Cell 2020, 32, 3978-4001. [CrossRef]

22. Delteil, A.; Gobbato, E.; Cayrol, B.; Estevan, J.; Michel-Romiti, C.; Dievart, A.; Kroj, T.; Morel, J.B. Several wall-associated kinases participate positively and negatively in basal defense against rice blast fungus. BMC Plant Biol. 2016, 16, 17. [CrossRef]

23. Li, H.; Zhou, S.Y.; Zhao, W.S.; Su, S.C.; Peng, Y.L. A novel wall-associated receptor-like protein kinase gene, OsWAK1, plays important roles in rice blast disease resistance. Plant Mol. Biol. 2009, 69, 337-346. [CrossRef] [PubMed]

24. Qi, H.; Zhu, X.; Guo, F.; Lv, L.; Zhang, Z. The Wall-Associated Receptor-Like Kinase TaWAK7D Is Required for Defense Responses to Rhizoctonia cerealis in Wheat. Int. J. Mol. Sci. 2021, 22, 5629. [CrossRef] [PubMed]

25. Malukani, K.K.; Ranjan, A.; Hota, S.J.; Patel, H.K.; Sonti, R.V. Dual Activities of Receptor-Like Kinase OsWAKL21.2 Induce Immune Responses. Plant Physiol. 2020, 183, 1345-1363. [CrossRef]

26. Brutus, A.; Sicilia, F.; Macone, A.; Cervone, F.; De Lorenzo, G. A domain swap approach reveals a role of the plant wall-associated kinase 1 (WAK1) as a receptor of oligogalacturonides. Proc. Natl. Acad. Sci. USA 2010, 107, 9452-9457. [CrossRef]

27. Dardick, C.; Schwessinger, B.; Ronald, P. Non-arginine-aspartate (non-RD) kinases are associated with innate immune receptors that recognize conserved microbial signatures. Curr. Opin. Plant Biol. 2012, 15, 358-366. [CrossRef]

28. Liu, X.; Zhu, X.; Wei, X.; Lu, C.; Shen, F.; Zhang, X.; Zhang, Z. The wheat LLM-domain-containing transcription factor TaGATA1 positively modulates host immune response to Rhizoctonia cerealis. J. Exp. Bot. 2020, 71, 344-355. [CrossRef]

29. Zhu, X.; Lu, C.; Du, L.; Ye, X.; Liu, X.; Coules, A.; Zhang, Z. The wheat NB-LRR gene TaRCR1 is required for host defence response to the necrotrophic fungal pathogen Rhizoctonia cerealis. Plant Biotechnol. J. 2017, 15, 674-687. [CrossRef] [PubMed]

30. Wan, J.; Zhang, S.; Stacey, G. Activation of a mitogen-activated protein kinase pathway in Arabidopsis by chitin. Mol. Plant Pathol. 2004, 5, 125-135. [CrossRef]

31. Miya, A.; Albert, P.; Shinya, T.; Desaki, Y.; Ichimura, K.; Shirasu, K.; Narusaka, Y.; Kawakami, N.; Kaku, H.; Shibuya, N. CERK1, a LysM receptor kinase, is essential for chitin elicitor signaling in Arabidopsis. Proc. Natl. Acad. Sci. USA 2007, 104, 19613-19618. [CrossRef] [PubMed]

32. Liu, B.; Li, J.F.; Ao, Y.; Qu, J.; Li, Z.; Su, J.; Zhang, Y.; Liu, J.; Feng, D.; Qi, K.; et al. Lysin motif-containing proteins LYP4 and LYP6 play dual roles in peptidoglycan and chitin perception in rice innate immunity. Plant Cell 2012, 24, 3406-3419. [CrossRef] [PubMed]

33. Cao, Y.; Liang, Y.; Tanaka, K.; Nguyen, C.T.; Jedrzejczak, R.P.; Joachimiak, A.; Stacey, G. The kinase LYK5 is a major chitin receptor in Arabidopsis and forms a chitin-induced complex with related kinase CERK1. eLife 2014, 3, 15. [CrossRef]

34. Shinya, T.; Yamaguchi, K.; Desaki, Y.; Yamada, K.; Narisawa, T.; Kobayashi, Y.; Maeda, K.; Suzuki, M.; Tanimoto, T.; Takeda, J.; et al. Selective regulation of the chitin-induced defense response by the Arabidopsis receptor-like cytoplasmic kinase PBL27. Plant J. 2014, 79, 56-66. [CrossRef]

35. Yamada, K.; Yamaguchi, K.; Shirakawa, T.; Nakagami, H.; Mine, A.; Ishikawa, K.; Fujiwara, M.; Narusaka, M.; Narusaka, Y.; Ichimura, K.; et al. The Arabidopsis CERK1-associated kinase PBL27 connects chitin perception to MAPK activation. EMBO J. 2016, 35, 2468-2483. [CrossRef] 
36. Zhu, X.; Yang, K.; Wei, X.; Zhang, Q.; Rong, W.; Du, L.; Ye, X.; Qi, L.; Zhang, Z. The wheat AGC kinase TaAGC1 is a positive contributor to host resistance to the necrotrophic pathogen Rhizoctonia cerealis. J. Exp. Bot. 2015, 66, 6591-6603. [CrossRef] [PubMed]

37. Guo, F.; Wu, T.; Shen, F.; Xu, G.; Qi, H.; Zhang, Z. The cysteine-rich receptor-like kinase TaCRK3 contributes to defense against Rhizoctonia cerealis in wheat. J. Exp. Bot. 2021,3, 15. [CrossRef]

38. Zhang, Z.Y.; Yao, W.L.; Dong, N.; Liang, H.X.; Liu, H.X.; Huang, R.F. A novel ERF transcription activator in wheat and its induction kinetics after pathogen and hormone treatments. J. Exp. Bot. 2007, 58, 2993-3003. [CrossRef]

39. Su, Z.; Bernardo, A.; Tian, B.; Chen, H.; Wang, S.; Ma, H.; Cai, S.; Liu, D.; Zhang, D.; Li, T.; et al. A deletion mutation in TaHRC confers Fhb1 resistance to Fusarium head blight in wheat. Nat. Genet. 2019, 51, 1099-1105. [CrossRef] [PubMed]

40. Wu, T.-C.; Zhu, X.-L.; LÜ, L.-J.; Chen, X.-Y.; Xu, G.-B.; Zhang, Z.-Y. The wheat receptor-like cytoplasmic kinase TaRLCK1B is required for host immune response to the necrotrophic pathogen Rhizoctonia cerealis. J. Integr. Agric. 2020, 19, 2616-2627. [CrossRef]

41. Wang, K.; Shao, Z.; Guo, F.; Wang, K.; Zhang, Z. The Mitogen-Activated Protein Kinase Kinase TaMKK5 Mediates Immunity via the TaMKK5-TaMPK3-TaERF3 Module. Plant Physiol. 2021, 15, 356. [CrossRef]

42. Livak, K.J.; Schmittgen, T.D. Analysis of relative gene expression data using real-time quantitative PCR and the 2(-Delta Delta C(T)) Method. Methods 2001, 25, 402-408. [CrossRef] [PubMed] 